\title{
Maternal and perinatal outcomes after bariatric surgery: a spanish multicenter study
}

\author{
Irene González ${ }^{1}$, Miguel A. Rubio ${ }^{2}$. Fernando Cordido ${ }^{3}$, Irene Bretón ${ }^{4}$, María J. \\ Morales $^{5}$, Nuria Vilarrasa ${ }^{6}$, Susana Monereo ${ }^{7}$, Albert Lecube ${ }^{8}$, Assumptas Caixàs ${ }^{9}$, \\ Irene Vinagre $^{10}$, Albert ${ }^{\text {Goday11-13 }}$,Pedro P. García-Luna ${ }^{14}$
}

\begin{abstract}
${ }^{1}$ Hospital Juan Ramón Jiménez, Huelva, Spain; ${ }^{2}$ Hospital Clínico San Carlos, IDISSC, Madrid, Spain; ${ }^{3}$ Hospital Universitario A Coruña, A Coruña, Spain; ${ }^{4}$ Hospital Universitario Gregorio Marañón, Madrid, Spain; ${ }^{5}$ Hospital del Meixoeiro, Vigo, Spain; ${ }^{6}$ Hospital Universitario de Bellvitge, Barcelona, Spain; ${ }^{7}$ Hospital Universitario Gregorio Marañón, Madrid, Spain; ${ }^{8}$ Hospital Universitari Arnau de Vilanova, Lleida, Spain; ${ }^{9}$ Hospital de Sabadell, Sabadell, Spain; ${ }^{10}$ Hospital Clínic i Universitari, Barcelona, Spain; ${ }^{11}$ Hospital del Mar, Barcelona, Spain; ${ }^{12}$ Departament de Medicina, Universitat Autònoma de Barcelona, Barcelona, Spain; ${ }^{13}$ CIBERobn (Centros de Investigación Biomédica en Red-CIBER, Physiopathology of Obesity and Nutrition), Instituto de Salud Carlos III, Madrid, Spain; 14 Hospital Universitario Virgen del Rocío, Sevilla, Spain;
\end{abstract}

\begin{abstract}
Background. Bariatric surgery (BS) has become more frequent among women of child-bearing age. Data regarding the underlying maternal and perinatal risks are scarce. The objective of this nationwide study is to evaluate maternal and perinatal outcomes after BS.

Methods. We performed a retrospective observational study of 168 pregnancies in 112 women who underwent BS in 10 tertiary hospitals in Spain over a 15-year period. Maternal and perinatal outcomes, including gestational diabetes mellitus (GDM), pregnancy-associated hypertensive disorders (PAHD), pre-term birth cesarean deliveries, small and large for gestational age births (SGA, LGA), still births, and neonatal deaths, were evaluated. Results were further compared according to the type of BS performed: restrictive techniques (vertical-banded gastroplasty, sleeve gastrectomy, and gastric banding), Roux-en-Y gastric bypass (RYGB), and biliopancreatic diversion (BPD).

Results. GDM occurred in five (3\%) pregnancies and there were no cases of PAHD. Women whose pregnancies occurred before 1 year after BS had a higher pre-gestational body mass index (BMI) than those who got pregnant 1 year after BS $\left(34.6 \pm 7.7\right.$ vs $\left.30.4 \pm 5.3 \mathrm{~kg} / \mathrm{m}^{2}, p=0.007\right)$. In pregnancies occurring during the first year after BS, a higher rate of stillbirths was observed compared to pregnancies occurring after this period of time (35.5 vs $16.8 \%$, $p=0.03$ ). Women who underwent BPD delivered a higher rate of SGA babies than women with RYGB or restrictive procedures (34.8, 12.7 , and $8.3 \%$, respectively).

Conclusions. Pregnancy should be scheduled at least 1 year after BS. Malabsorptive procedures are associated to a higher rate of SGA births.
\end{abstract}

Keywords

Bariatric surgery; Pregnancy; Obesity; Adverse maternal and neonatal outcomes; Perinatal outcomes 


\section{Introduction}

The increasing prevalence of obesity has currently reached epidemic proportions [1]. In Spain, recent data have calculated a prevalence of obesity of $21.4 \%$ in women [2], which is similar to what has been reported in Mediterranean regions such as Italy and Portugal, and in other countries like Poland, Czech Republic, Romania, and Albania [3].

Besides the negative health impact of obesity itself, it is a risk factor for adverse maternal and perinatal outcomes, such as gestational diabetes mellitus (GDM), pregnancy-associated hypertensive disorders (PAHD), macrosomia, cesarean deliveries, pre-term birth, fetal malformations, and perinatal mortality [4]. Furthermore, obesity complicates recovery from epidural or general anesthesia, increases the overall period of labor, and increases the probability of post-partum hemorrhage, venous thrombosis, and pulmonary thromboembolism [5]. Thus, obese women represent an especially high-risk group of patients.

Weight loss has proved to reduce these adverse maternal and perinatal outcomes as well as secondary infertility, so it could be hypothesized that bariatric surgery (BS) would also achieve the same positive results. Nowadays, approximately half of the patients who undergo bariatric procedures are women of child-bearing age. This has raised concerns among obstetricians, endocrinologists, and surgeons regarding its consequences for future pregnancies and their outcomes. Moreover, a correct nutritional intervention is crucial during pregnancy [6], but the greatest weight loss after BS occurs during the first 12-18 postoperative months [7]. Consequently, this period would theoretically convey an increased risk of malnutrition for both the mother and the fetus.

There are few studies that evaluate maternal and perinatal outcomes after BS, and there are currently no clinical practice guidelines that address this issue. The Group of Obesity of the Spanish Society of Endocrinology and Nutrition (GOSEEN) reviewed a cohort of post-bariatric pregnant women in order to evaluate maternal and perinatal outcomes after BS.

\section{Methods}

\section{Study Population}

We conducted a multicenter retrospective observational study of all pregnancies, both singleton and multiple, in women who had previously undergone BS during the period of January 1998 through December 2012 in 10 tertiary reference public hospitals in Spain. Data was collected by extensively reviewing medical charts. Pre-gestational variables included the following: maternal age and pregestational body weight and body mass index (BMI), the type of BS performed, and time in months from BS to pregnancy. Pre-gestational body weight was considered as the one registered in the last prepregnancy visit or the one referred by the patient herself; its corresponding BMI was calculated accordingly $\left(\mathrm{kg} / \mathrm{m}^{2}\right)$. The type of BS was grouped into three categories: restrictive techniques, which included adjustable gastric band, vertical band, and sleeve gastrectomy; malabsorptive procedures, which included Scopinaro diversion and duodenal switch; and mixed techniques, which mainly comprised Roux-en-Y gastric bypass (RYGB). Table 1 shows the distribution of patients according to the type of BS performed. 
Table 1 Number (and percentages of total) of patients included in our study, according to the type of bariatric surgery performed

\begin{tabular}{llll}
\hline & Laparoscopic & Open surgery & Total \\
\hline & & & \\
Restrictive procedures & $25(14.9)$ & $7(4.2)$ & $32(19)$ \\
$\quad$ Sleeve gastrectomy & $22(13.1)$ & $0(0)$ & $22(13.1)$ \\
Vertical-banded gastroplasty & $0(0)$ & $7(4.2)$ & $7(4.2)$ \\
Gastric banding & $3(1.8)$ & $0(0)$ & $3(1.8)$ \\
Roux-en-Y gastric bypass & $60(35.7 \%)$ & $20(11.9 \%)$ & $80(47.6 \%)$ \\
Biliopancreatic diversion & $17(10.2 \%)$ & $39(23.2 \%)$ & $56(33.3 \%)$ \\
& & & \\
\hline
\end{tabular}

Levels of vitamins, including vitamins $\mathrm{A}, \mathrm{D}, \mathrm{E}, \mathrm{B}_{12}$, and folic acid, and some micronutrients such as iron, calcium, and zinc were monitored during the course of pregnancy. Supplemental intake of all vitamins and micronutrients was also registered.

Development of pregnancy-associated complications such as GDM, hypertension, and preeclampsia was recorded. Women were screened for GDM based on the 3rd Workshop Conference on Gestational Diabetes Mellitus [8].

Regarding perinatal outcomes, variables collected included the type of delivery performed (normal birth, dystocia delivery, elective, or urgent cesarean section), the need for urgent blood transfusions or antibiotic therapy, gestational age, birth weight, fetal malformations, stillbirths, and neonatal mortality. A compound variable for the diagnosis of adverse maternal outcomes was elaborated, which was considered when at least one of the following was present: GDM, PAHD, pre-term birth, and intrauterine growth restriction.

Gestational age was calculated according to weeks of amenorrhea and ultrasound findings. Birth weight was correlated to gestational age as a proxy for fetal growth and was compared to fetal growth curves obtained by Carrascosa et al., which are adapted to Spanish population [9]. Small for gestational age (SGA) was considered when birth body weight was below the third percentile, and large for gestational age (LGA) was considered when it was above percentile 90. Additionally, low birth weight was considered if it was $<2,500 \mathrm{~g}$ and macrosomia was considered if $>4,000 \mathrm{~g}$. Another compound variable was created according to the presence of an adverse fetal outcome, which was considered when one of the following occurred: SGA, LGA, fetal malformations, need for incubator care, severe neonatal disease, fetal stress, or neonatal mortality.

This study was approved by the Ethics Committees of each hospital and was in compliance with the Helsinki Declaration. All patients signed a written informed consent in which it was specified that data collected from their medical charts could be potentially used in an anonymous way for investigation and publication.

\section{Statistical Analysis}

IBM SPSS Statistics version 15.0 (Chicago, IL, USA) was used for statistical analysis. For descriptive data, results were expressed as mean \pm standard deviation and median (interquartile range) for normally and not-normally distributed continuous variables, respectively. Categorical variables were summarized as frequencies and percentages. Comparison of continuous variables between groups was performed using Mann Whitney $T$ or $U$ tests, and analysis of variance or Kruskal-Wallis tests, as appropriate. Comparison between categorical groups was assessed with chi-square test and Fisher's exact test as required. Pre- 
gestational features were examined for their influence on maternal and perinatal adverse outcomes using binary logistic regression analysis. Significance was considered at $p<0.05$.

\section{Results}

A total of 168 pregnancies (165 singleton and 3 multiple pregnancies) were reviewed from a total of 112 women who underwent BS during the period of study. Mean age at gestation was $33.2 \pm 4.2$ years, mean BMI prior to BS was $49.3 \pm 8.2 \mathrm{~kg} / \mathrm{m}^{2}$, mean pre-gestational BMI was $31.2 \pm 6.0 \mathrm{~kg} / \mathrm{m}^{2}$, and median time from BS to pregnancy was 30.7 (2-134) months. Of cases, $19 \%$ underwent restrictive techniques, $46.7 \%$ RYGB, and $33.3 \%$ underwent a malabsorptive procedure.

Overall, $81 \%$ of women reported intake of multivitamins during pregnancy. Specifically, $73.8 \%$ took iron supplements, $45.9 \%$ calcium, $47.6 \%$ 25-hydroxyvitamin $\mathrm{D}, 47 \%$ vitamin $\mathrm{B}_{12}, 7.7 \%$ vitamin $\mathrm{A}$, $4.8 \%$ vitamin E, $4.2 \%$ zinc, and $0.6 \%$ vitamin $\mathrm{K}$, according to the results observed in laboratory analysis. There were 9 cases $(5.4 \%)$ of folic acid deficiency during pregnancy. In one case, this was followed by a spontaneous miscarriage due to a defective embryological development, and in another case, the fetus presented with pyelectasis plus agenesis of corpus callosum. All women underwent triple screening, and pathological results were only observed in two cases: one case turned into a spontaneous miscarriage at 12 weeks, and in the other one, the mother decided to voluntarily end her pregnancy.

Liquid oral supplements were required in three patients $(1.8 \%)$ due to severe hyperemesis gravidarum and risk of malnutrition.

In the majority of cases, there were no medical or surgical complications during pregnancy. Only five patients $(3 \%)$ developed GDM, but none of them suffered from PAHD; however, $3.6 \%$ of women had been diagnosed with essential hypertension before pregnancy.

If we consider the compound variable for maternal complications, adverse outcomes were identified in $6.7 \%$ of pregnancies. We did not identify an association between maternal age, pre-gestational BMI, or time from BS to pregnancy with maternal adverse events.

From a total of 132 full-term pregnancies, there were 134 newborns, with a mean gestational age of $38.9 \pm 2.6$ weeks. Pre-term births were $10.5 \%$ and there were 34 cases $(20.2 \%)$ of stillbirths. Vaginal delivery was accomplished in $79.8 \%$ of cases $(61.9 \%$ spontaneous eutocic delivery, $11.9 \%$ induced eutocic delivery, and $5.9 \%$ labor dystocia) and a cesarean section was required in $19.4 \%$ of cases ( $11.9 \%$ primary elective, $7.5 \%$ urgent). We did not observe an association between the type of surgery performed and the number of cesarean sections or the number of pre-term births (Table 2). 
Table 2 Maternal and perinatal outcomes according to the type of bariatric surgery performed

\begin{tabular}{|c|c|c|c|c|}
\hline Variables & Restrictive procedures & RYGB & BPD & $p$ value \\
\hline Number of patients & $32(19)$ & $80(47.6)$ & $56(33.3)$ & \\
\hline Fetal loss & $8(25)$ & $16(20)$ & $10(17.9)$ & 0.723 \\
\hline Pre-surgical BMI (kg/m²) & $45.7 \pm 7.2$ & $46.7 \pm 6.4$ & $53.3 \pm 8.7$ & 0.003 \\
\hline$\% \mathrm{WL}$ & $35.1 \pm 11.4$ & $32.9 \pm 8.3$ & $39.7 \pm 9.1$ & 0.008 \\
\hline Pre-gestational BMI $\left(\mathrm{kg} / \mathrm{m}^{2}\right)$ & $31.3 \pm 5.6$ & $30.7 \pm 4.9$ & $32.2 \pm 6.3$ & 0.266 \\
\hline Pre-term birth & $4 / 24(16.7)$ & $8 / 63(12.7)$ & $3 / 46(6.5)$ & 0.394 \\
\hline \multicolumn{5}{|l|}{ Type of delivery } \\
\hline Cesarean & $5 / 23(21.7)$ & $15 / 64(23.4)$ & $6 / 46(13.1)$ & 0.382 \\
\hline Vaginal & $18 / 23(78.3)$ & $49 / 64(76.6)$ & $40 / 46(86.9)$ & \\
\hline SGA & $2 / 24(8.3)$ & $8 / 63(12.7)$ & $16 / 46(34.8)$ & 0.005 \\
\hline Combined perinatal adverse outcome & $8 / 25(32)$ & $26 / 65(40)$ & $16 / 44(36.4)$ & 0.771 \\
\hline Combined maternal adverse outcome & $0 / 25(0)$ & $8 / 65(12.3)$ & $1 / 44(2.3)$ & 0.06 \\
\hline
\end{tabular}

\begin{abstract}
Values show mean \pm SD or number of patients and percentages $(\%)$. $p$ values for comparisons using chi-square analysis for categorical variables and analysis of variance for continuous variables. For explanation of combined perinatal and maternal adverse outcome, see main text $R Y G B$ Roux-en-Y gastric bypass, $B P D$ biliopancreatic diversion, $B M I$ body mass index, $S G A$ small for gestational age birth, \%WL percentage weight loss (minimum weight achieved before pregnancy)
\end{abstract}

Mean birth weight was 3,008 $\pm 599 \mathrm{~g} ; 19.6 \%$ was SGA, $6.8 \%$ was LGA, and 4 (3\%) newborns were considered macrosomic. Low birth weight was recorded in $15.7 \%$ of newborns, including 4 cases from twin gestations. Regarding fetal adverse outcomes, there were 3 cases of fetal malformations: one child with cavovarus foot, one with aortic coarctation, and another one with fetal pyelectasis plus agenesis of corpus callosum. Furthermore, there were $17.9 \%$ fetal losses (6 stillbirths, 2 ectopic pregnancies, and 22 spontaneous abortions) and 4 cases of voluntary interruption of pregnancy.

In comparison to women whose pregnancies occurred 1 year after BS, women whose pregnancies occurred before this period exhibited a significantly higher BMI $\left(34.6 \pm 7.7 \mathrm{~kg} / \mathrm{m}^{2}\right)$ and an increased rate of fetal loss $(35.5 \%)$. However, there were no differences between both groups in the rate of perinatal adverse outcomes when the compound variable was considered (42.9 vs $36.6 \%, p=0.766$ ) (Table 3 ). 
Table 3 Maternal and perinatal adverse outcomes according to time of pregnancy after bariatric surgery (before or after 1 year)

\begin{tabular}{llll}
\hline Variable & $\begin{array}{l}<\text { year } \\
n=31\end{array}$ & $\begin{array}{l}>1 \text { year } \\
n=137\end{array}$ & $p$ value \\
\hline & & & 0.484 \\
Age & $32.8 \pm 4.7$ & $33.3 \pm 4.1$ & 0.03 \\
Fetal loss & $11 / 31(35.5)$ & $23 / 137(16.8)$ & 0.007 \\
Pre-gestational BMI $\left(\mathrm{kg} / \mathrm{m}^{2}\right)$ & $34.6 \pm 7.7$ & $30.4 \pm 5.3$ & 1 \\
Pre-term birth & $2 / 20(10)$ & $13 / 114(11.4)$ & 0.543 \\
Type of delivery & & & 0.543 \\
$\quad$ Cesarean & $5 / 20(25)$ & $21 / 114(18.4)$ & 0.74 \\
$\quad$ Vaginal & $15 / 20(75)$ & $92 / 114(80.7)$ & 1 \\
SGA & $5 / 20(25)$ & $21 / 114(18.4)$ & $41 / 114(35.9)$ \\
Combined perinatal adverse outcome & $9 / 21(42.9)$ & $8 / 114(7.1)$ & \\
Combined maternal adverse outcome & $1 / 21(4.8)$ & & \\
\end{tabular}

\begin{abstract}
Values show mean $\pm \mathrm{SD}$ or number of patients and percentages (\%). $p$ values for comparisons using chi-square analysis for categorical variables and analysis of variance for continuous variables. For explanation of combined perinatal and maternal adverse outcome, see main text

$R Y G B$ Roux-en-Y gastric bypass, $B P D$ biliopancreatic diversion, $B M I$ body mass index, $S G A$ small for gestational age birth
\end{abstract}

Binary logistic regression analysis did not show an association between maternal age, gestational BMI, or time from BS to pregnancy (considered as $<$ or $>1$ year) and an increased risk of adverse outcomes (Table 4). Evaluation of obstetric outcomes according to the type of BS performed revealed that women who underwent malabsorptive procedures delivered more SGA babies (34.8\%), compared to women who underwent restrictive techniques $(8.3 \%)$ or RYGB (12.7\%) (Table 2).

Table 4 Results for binary logistic regression analysis, evaluating the influence of different characteristics on maternal and perinatal adverse outcomes

\begin{tabular}{lllll}
\hline & & OR & $95 \% \mathrm{CI}$ & $p$ \\
\hline \multirow{3}{*}{ Adverse maternal outcome } & Maternal age & & & \\
& Pre-gestational BMI & 1.939 & $0.496-7.576$ & 0.266 \\
& Time from BS to pregnancy & 0.868 & $0.222-3.386$ & 0.558 \\
Adverse perinatal outcome & 0.650 & $0.077-5.487$ & 0.569 \\
& Maternal age & 1.277 & $0.627-2.600$ & 0.623 \\
& Pre-gestational BMI & 0.529 & $0.258-1.085$ & 0.117 \\
& Time from BS to pregnancy & 1.299 & $0.504-3.345$ & 0.766 \\
& & & \\
\hline
\end{tabular}

Time from BS to pregnancy was considered a categorical variable $(<$ or $>1$ year $)$

OR odds ratio, CI $95 \% 95 \%$ confidence interval, $p$ p value for the Wald statistic, BMI body mass index, BS bariatric surgery 


\section{Discussion}

Our study evaluates a large retrospective and multicenter cohort of pregnancies in women after three types of bariatric procedures. One of the most relevant findings is that women who got pregnant during the first year after BS seemed to have a higher frequency of fetal loss, in comparison to those who got pregnant after 1 year following BS. Moreover, women who underwent malabsorptive procedures, such as BPD, gave birth to more SGA newborns, in comparison to women who underwent other types of techniques. However, the prevalence of GDM and PAHD was almost anecdotic.

Regarding pre-gestational BMI, other reports have observed that women usually get pregnant at a mean BMI between 25.1 and $30.6 \mathrm{~kg} / \mathrm{m}^{2}[10,11,12,13]$, but in our series, the majority of women who became pregnant were still under the category of obesity $\left(31.2 \pm 6.0 \mathrm{~kg} / \mathrm{m}^{2}\right)$. On the other hand, in our group, mean time from BS to pregnancy was 30 months. This is in accordance with current recommendations, which suggest a minimum waiting period of 1 year after BS before becoming pregnant. We found that women who got pregnant before 1 year after BS presented a higher rate of fetal loss, in comparison to women whose pregnancy occurred after this period of time (35.5 vs $16.3 \%)$. This difference has not been acknowledged in other previous studies [14, 15, 16, 17]. A possible explanation may rely on the higher pre-gestational BMI that women in our series presented; however, it is also plausible that a greater catabolism in the setting of significant weight loss during the first postoperative year contributed to the higher rates of fetal loss observed. Therefore, it would seem reasonable and prudent to recommend waiting a minimum period of 1 year before planning future pregnancies.

We remark the low prevalence of obstetric comorbidities observed in our study population. Specifically, regarding GDM, it has been well-demonstrated that age, BMI, and multiparity are important risk factors for its development $[18,19,20]$, and a recent publication estimated a prevalence of $8.8-$ $11.6 \%$, depending on the criteria used [21]. However, our study agrees with other previous reports in which lower rates of GDM were accounted in obese women whose pregnancy followed BS, in comparison to women whose obesity was only managed conservatively [11,22]. The reason for the low prevalence of GDM observed in our study deserves further explanation. Insulin resistance is indeed decreased after BS due to significant weight loss [23] and a healthier lifestyle, and this would therefore be associated to a reduced risk of developing GDM. Nevertheless, the oral glucose tolerance test (OGTT) should be well standardized and validated to be able to confidently diagnose GDM in women who underwent BS because gastric emptying may be altered, so using this method may underestimate the prevalence of GDM. Additionally, an improved adherence to lifestyle modifications, as well as optimal serum levels of 25-hydroxyvitamin D following BS may also contribute to ameliorating insulin sensitivity and prevent the development of GDM [24].

Regarding hypertensive disorders, although results are less consistent, most studies have observed a reduced rate of these conditions in post-bariatric pregnancies, compared to pregnancies in non-operated BMI-matched obese women [25]; specifically, PAHD have been estimated to occur in around $4 \%$ of pregnancies in our country [20]. No cases of PAHD were documented in our study population, even though it is well-known that these conditions are frequently observed in overweight and obese pregnant women, especially during the first term [26].

If we now turn to the evaluation of nutrient deficiencies, very few studies have specifically addressed this issue in pregnancies occurring after BS. Our study showed that the most frequent nutrient deficiency was that of iron and/or ferritin, followed by vitamin $\mathrm{D}$ and vitamin $\mathrm{B}_{12}$, in agreement with other studies [12]. This probably reflects the most prevalent deficiencies which normally occur after BS and which may appear during pregnancy despite previous adequate supplementation. When evaluating these results, we must bear in mind that most pregnant women regularly take prescribed multivitamins, and many of them also take specific nutrient supplements. However, in the particular case of 25-hydroxyvitamin D, although it is routinely prescribed after BS, it is not regularly taken by the general population. Several studies have pointed out that low levels of 25 -hydroxyvitamin $\mathrm{D}(<20 \mathrm{ng} / \mathrm{dL})$ are associated to an increased rate of pre-term birth and cesarean delivery [24], highlighting the importance of maintaining adequate levels of this nutrient during pregnancy. 
In our series, there were three cases of hyperemesis gravidarum, but all were adequately managed in a conservative way, using oral liquid supplements in order to avoid the risk of malnutrition. Even though gastrectomy itself may entail a higher rate of vomiting, the rate of this adverse event observed in our cohort was similar to what has been described in normal pregnancies $(0.5-2 \%)$ [25].

We identified a prevalence of fetal loss of $17.9 \%$. This should not be considered a negative outcome, since rates of around 24 and $30 \%$ have been described in normal pregnancies [20] and pregnancies following BS [11, 12], respectively. Regarding vaginal C-sections, we have not found that rates in our series differed significantly from what has been steadily reported in our country over the last decade [20, 26].

Some authors have already suggested an increased rate of spontaneous abortion, pre-term deliveries, and premature births in pregnancies occurring in the early post-bariatric period, while successful pregnancies have been documented within 1 or 2 years after BS [27]. There is still insufficient evidencederived data to confidently recommend an ideal timing for pregnancy after BS, but waiting a period of at least 1 year seems a reasonable approach, according to the results derived from our study [28]. Therefore, it seems prudent to recommend the use of contraceptive methods during at least the first postoperative year after BS.

Another remarkable finding of our study is that the frequency of SGA newborns was higher in women who underwent BPD. Malnutrition and malabsorption following these types of procedures may be a possible explanation for this outcome. In this regard, in a systematic review of pregnancies following BS, there were no differences in the rate of SGA between women who underwent restrictive and mixed techniques. In fact, rates of SGA in women who underwent restrictive techniques and gastric bypass were in the range of what has been reported in the general population (around 7-8\%) [20]. However, in patients who underwent BPD, a higher rate of SGA was documented [29, 30]. Another retrospective study, which included a total of 2,562 pregnancies following BS, concluded that women who had previously undergone a bariatric procedure had a higher risk of spontaneous or induced pre-term birth, as well as an increased rate of SGA [31]; therefore, these women should be considered as a high-risk group during pregnancy.

Nevertheless, the hypothesis that a lower birth weight may convey a negative impact in the offspring's development has not been fully elucidated. The study by Smith et al. [32] evaluated children aged 2.526 years born before and after maternal BS (in this case, BPD). They found improvements in cardiometabolic risk factors, sustained into adolescence, despite the fact that birth weight in these children was lower in comparison to children born from morbidly obese women. In this regard, data on the long-term follow-up of the offspring of women who underwent BPD may suggest that an improved intrauterine environment could play a major role in minimizing epigenetic influences on obesity and other metabolic disorders in adolescence and adulthood [32].

\section{Conclusions}

Overall, pregnancies occurring after restrictive or mixed techniques are not associated to an increased risk of maternal or perinatal adverse outcomes, nor nutritional deficiencies. In fact, pregnancy-associated comorbidities such as GDM and PAHD are uncommon. Pregnant women who underwent malabsorptive procedures such as BPD exhibit a higher probability of having SGA offspring, and should therefore be considered as high-risk patients. Pregnancies occurring before 1 year after BS are associated to an increased rate of perinatal adverse outcomes, mainly spontaneous abortions. Therefore, scheduling pregnancy after at least 1 year after BS seems a practical recommendation to give to our post-bariatric female patients. 


\section{Acknowledgments}

We thank The Group of Obesity of the Spanish Society of Endocrinology and Nutrition (GOSEEN) for participating and providing data for this study, and Dr. Ana M Ramos-Leví for her valuable help in the preparation of this manuscript.

\section{Conflict of Interest}

The authors declare that they have no conflict of interest.

\section{References}

1. Finucane MM, Stevens GA, Cowan MJ, et al. National, regional, and global trends in bodymass index since 1980: systematic analysis of health examination surveys and epidemiological studies with 960 country-years and 9.1 million participants. Lancet. 2011;377:557-67.

2. Gutiérrez-Fisac JL, Guallar-Castillón P, León-Muñoz LM, et al. Prevalence of general and abdominal obesity in the adult population of Spain. 2008-2010: the ENRICA study. Obes Rev. 2012;13:388-92.

3. Berghöfer A, Pischon T, Reinhold T, et al. Obesity prevalence from a European perspective: a systematic review. BMC Public Health. 2008;8:200-10.

4. Nuthalapaty FS, Rouse DJ. The impact of obesity on obstetrical practice and outcome. Clin Obstet Gynecol. 2004;47:898-913.

5. ACOG practice bulletin (developed by the ACOG committee on Practice Bulletins). Bariatric surgery and pregnancy. Obstet Gynecol. 2009;113:1405-13.

6. Southern California Evidence-based Practice Center (EPC). Bariatric surgery in women of reproductive age: special concerns for pregnancy. Evidence report/technology assessment number 169.AHRQ publication no. 08-E013; 2008.

7. Courcoulas AP, Christian NJ, Belle SH, et al. Weight change and health outcomes at 3 years after bariatric surgery among individuals with severe obesity. JAMA. 2013;310:2416-25.

8. Metzger BE. Summary and recommendations of the Third International Workshop-conference on Gestational Diabetes Mellitus. Diabetes. 1991;40 suppl 2:197-201.

9. Carrascosa Lezcano A, Ferrández Longás A, Yeste Fernández D, et al. Estudio transversal español de crecimiento 2008. Parte I: valores de peso y longitud de peso y longitud en recién nacidos de 26-42 semanas de edad gestacional. An Pediatr (Barc). 2008;68:544-51.

10. Sheiner E, Balaban E, Dreiher J, et al. Pregnancy outcome in patients following different types of bariatric surgeries. Obes Surg. 2009;19:1286-92.

11. Aricha-Tamir B, Weintraub AY, Levi I, et al. Downsizing pregnancy complications: a study of paired pregnancy outcomes before and after bariatric surgery. Surg Obes Relat Dis. 2012;8:434-9.

12. Bebber FE, Rizzolli J, Schaan-Casagrande DS, et al. Pregnancy after bariatric surgery: 39 pregnancies follow-up in a multidisciplinary team. Obes Surg. 2011;21:1546-51.

13. Dell'Agnolo CM, De Barros Carvalho MD, Pelloso SM. Pregnancy after bariatric surgery: implications for mother and newborn. Obes Surg. 2011;21:699-706

14. Sheiner E, Edri A, Balaban E, et al. Pregnancy outcome of patients who conceive during or after the first year following bariatric surgery. Am J Obstet Gynecol. 2011;204:50.e1-e6.

15. Wax JR, Cartin A, Wolff R, et al. Pregnancy following gastric bypass for morbid obesity: effect of surgery-to-conception interval on maternal and neonatal outcomes. Obes Surg. 2008;18:1517-21.

16. Patel JA, Patel NA, Thomas RL, et al. Pregnancy outcomes after laparoscopic Roux-en-Y gastric bypass. Surg Obes Relat Dis. 2008;4:39-45.

17. Sapre N, Munting K, Pandita A, et al. Pregnancy following gastric bypass surgery: what is the expected course and outcome? N Z Med J. 2009;122:33-42.

18. Pérez-Ferre N, Fernández D, Torrejón MJ, et al. Effect of lifestyle on the risk of gestational diabetes and obstetric outcomes in immigrant Hispanic women living in Spain. J Diabetes. 2012;4:432-8

19. Ramos-Leví AM, Pérez-Ferre N, Fernández MD, et al. Risk factors for gestational diabetes mellitus in a large population of women living in Spain: implications for preventative strategies. Int J Endocrinol. 2012;2012:312529. 
20. Duran A, Sáenz S, Torrejón MJ, et al. Introduction of IADPSG criteria for the screening and diagnosis of gestational diabetes mellitus results in improved pregnancy outcomes at a lower cost in a large cohort of pregnant women: The St. Carlos Gestational Diabetes Study. Diabetes Care. 2014 [Epub ahead of print].

21. Ricart W, López J, Mozas J, et al. Spanish group for the Study of the impact of Carpenter and Coustan GDM thresholds. Potential impact of American Diabetes Association (2000) criteria for diagnosis of gestational diabetes mellitus in Spain. Diabetologia. 2005;48:1135-41.

22. Lesko J, Peaceman A. Pregnancy outcomes in women after bariatric surgery compared with obese and morbidly obese controls. Obstet Gynecol. 2012;119:547-54.

23. Rao RS, Yanagisawa R, Kini S. Insulin resistance and bariatric surgery. Obes Rev. 2012;13:316-28.

24. Perez-Ferre N, Torrejon MJ, Fuentes M, et al. Association of low serum 25-hydroxyvitamin D levels in pregnancy with glucose homeostasis and obstetric and newborn outcomes. Endocr Pract. 2012;18:676-84.

25. Klebanoff MA, Koslowe PA, Kaslow R, et al. Epidemiology of vomiting in early pregnancy. Obstet Gynecol. 1985;66:612-6.

26. Martínez-Frías ML, Bermejo E, Rodríguez-Pinilla E, et al. Secular evolution and evolution according to autonomous communities of the frequency of fertility treatments, multiple deliveries and cesarean sections in Spain [in Spanish]. Med Clin (Barc). 2005;124:132-9.

27. Robinson HE, O'Connell CM, Joseph KS, et al. Maternal outcomes in pregnancies complicated by obesity. Obstet Gynecol. 2005;106:1357-64.

28. García-Luna PP, Gonzalez-Navarro I. Pregnancy after bariatric surgery: what should we tell our patients? Endocrinol Nutr. 2014;61(2):65-7.

29. Maggard MA, Yermilov I, Li Z, et al. Pregnancy and fertility following bariatric surgery: a systematic review. JAMA. 2008;300:2286-96.

30. Marceau P, Kaufman D, Biron S, et al. Outcome of pregnancies after biliopancreatic diversion. Obes Surg. 2004;14(3):318-24.

31. Roos N, Neovius M, Cnattingius S, et al. Perinatal outcomes after bariatric surgery: nationwide population based matched cohort study. BMJ. 2013;347:f6460.

32. Smith J, Cianflone K, Biron S, et al. Effects of maternal surgical weight loss in mothers on intergenerational transmission of obesity. J Clin Endocrinol Metab. 2009;94:4275-83. 